\title{
Scrambled Voice Frequency Signal Transmission in an Amplify and Forward Relaying Based STBC Encoded Cooperative MIMO-OFDM System
}

\author{
K. J. A. Chisty ${ }^{1}$, S. M. A. Islam ${ }^{2}$, S. E. Ullah ${ }^{3}$ and S. R. Sabuj ${ }^{4}$ \\ ${ }^{1,2}$ Department of Physics, Jahangirnagar University \\ ${ }^{3}$ Department of APEE, University of Rajshahi, \\ ${ }^{4}$ Department of EEE, Bangladesh University \\ ${ }^{1}$ kja_chisty@yahoo.com, 2azharphyd@yahoo.com, \\ 3enayet67@yahoo.com, ${ }^{4}$ saifuriict@gmail.com
}

\begin{abstract}
In this paper, a comprehensive study has been made on performance assessment of a single relayed cooperative wireless communication system under implementation of OFDM aided MIMO technology. This paper provides a new insight on system performance with proposed STBC encoded MIMO-OFDM radio interface technology, four channel equalization techniques (ZF, MMSE, ZF-SIC and MMSE-SIC) and three digital modulation schemes (BPSK, QPSK and QAM). Simulation results show that the STBC encoded single relayed MIMO-OFDM cooperative wireless communication system with BPSK and MMSE-SIC outperforms as compared to other digital modulations and channel equalization schemes on scrambled voice frequency signal transmission.
\end{abstract}

Keywords: Cooperative communication, Orthogonal frequency division multiplexing, Amplify and forward, Minimum mean square error and Zero forcing

\section{Introduction}

The increasing demands for providing wireless multimedia like audio, video, image and data as well as interactive high speed internet services are fueling intensive research efforts to materialize the vision of future generation wireless communication. In cellular mobile devices as well as in wireless sensor and ad-hoc networks, it is keenly noticeable that the deployment of MIMO technology at mobile user side is not always feasible mainly due to size and power constraints [1]. An innovative approach to harness the spatial diversity without deploying multiple antennas is cooperative diversity /cooperative communication. In this research work, two transmitting and two receiving antennas have been deployed with harnessing spatial diversity under cooperative communication.

Cooperative communication technique takes the advantage of broadcasting nature of wireless transmission through effective creation of a virtual antenna array in cooperating nodes [2]. Various types of cooperative communication have been proposed to achieve the goal such as amplify and forward (AF), decode and forward (DF), fixed relaying (FR), selection relaying (SR), coded cooperation (CC) and compress and forward (CF) were introduced in [3-6] respectively. The benefit of AF relay protocol is its simplicity and low cost implementation. DF relays which decode and possibly re-encode the information before transmission it and AF relays which forward the signal without hard decoding [7]. D. Haque et al. presented for a concatenated interleaved Forward Error Correction (FEC) scheme on the 
transmission of a voice signal. The simulation results showed that the implementation of interleaved Reed-Solomon code with Convolution code BPSK modulation is highly effective to combat inherent interference in the communication system and the transmitted voice signal is found to retrieve well under noisy circumstance [8]. M. G. Rashed et al. looked into the impact of FEC codes namely Cyclic Redundancy Code (CRC) and Convolution Code on the performance of OFDM wireless communication system for speech signal transmission over both AWGN and fading (Rayleigh and Rician) channels in term of BER. In the fading channels, it is found via computer simulation that the performance of the Convolution interleaved based OFDM systems outperform than that of CRC interleaved OFDM system as well as uncoded OFDM channels [9].

In this paper, a simulation model for cooperative MIMO-OFDM with single relay implementation of AF scheme has been established. The revolutionary concept of space time block coding (STBC) introduced in the last decade that the deployment of multiple antennas at the transceiver has been included cooperative MIMO-OFDM scheme. The performance of the system for the zero-forcing (ZF) and minimum mean square error (MMSE), successive interference cancellation (SIC) based minimum mean square error (MMSE-SIC) and zero forcing (ZF-SIC) has been evaluated in terms of BER and voice frequency signal transmission.

\section{System Model}

A cooperative MIMO-OFDM wireless communication system with single relay is shown in Fig. 1. It consists of two users, one user acts as the source while the other user serves as the receiver and the two users may interchange their information as source and receiver at different instants of time. Here, the input binary data is obtained from a portion of recorded audio (voice) signal. A simple convolution encoder of rate 1/2 is used in channel coding scheme to meet up such requirement. Digitally modulated signal using BPSK, QPSK, 16PSK and 64PSK are obtained by the conversion interleaved binary data. These data are rearranged according to the Space Time Block Code (STBC).

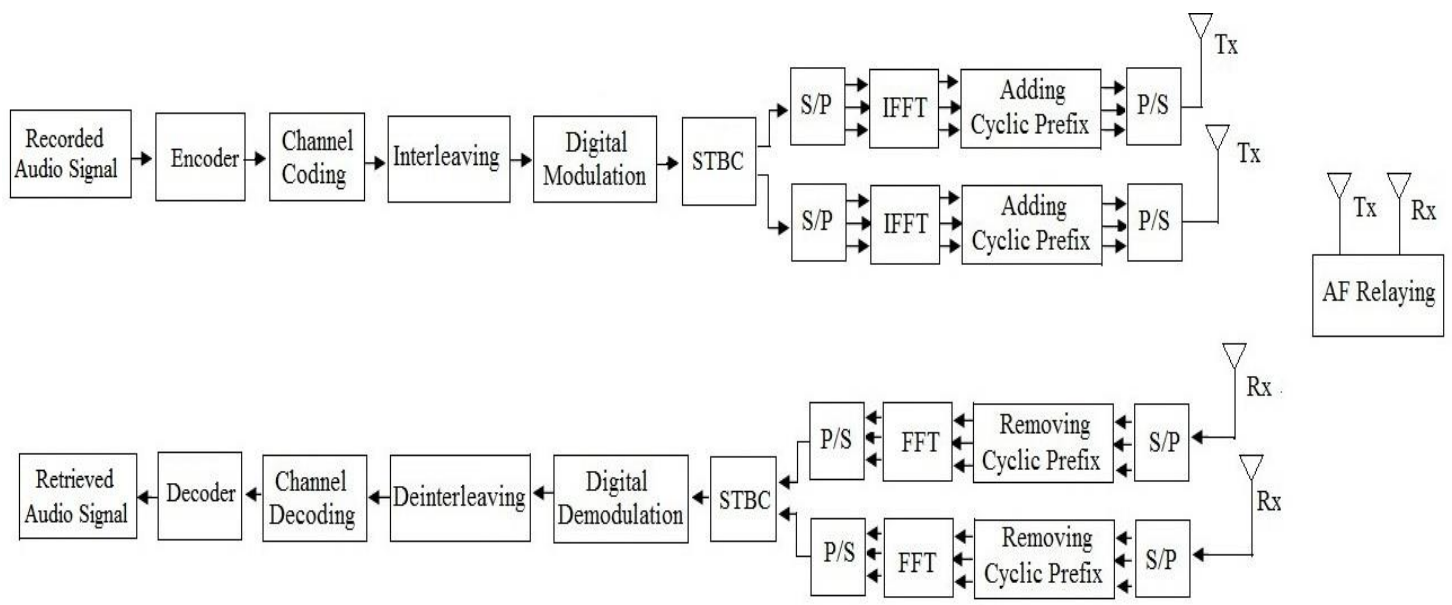

Figure 1. Block Diagram of Single Amplify and Forward Relaying based STBC Encoded Cooperative MIMO-OFDM System

The first antenna transmits the original data and negative second conjugate data while the second antenna transmits the conjugate original data and second data $[10,11]$. In each section 
of transmitting antenna, the data are passed through serial to parallel converter $(\mathrm{S} / \mathrm{P})$ and then transformed into time domain signal using inverse fast Fourier transform (IFFT). A cyclic prefix is then added that is a copy of the last elements of the frame. Information symbols are simultaneously transmitted through each transmitting antenna after parallel to serial conversion $(\mathrm{P} / \mathrm{S})$. Signals are received at the receiving end in two phases (phase I - direct link and phase II - relay link). The data are transferred from source to destination and source to relay directly in case of phase I. On the contrary, in phase II, data are transferred from relay to destination. Each cooperative relay has to amplify its received signal and then has to forward its amplified form through a wireless channel to the destination. At the destination section, the received signals are afterward passed through the process of OFDM demodulation, digital demodulation, deinterleaving, channel decoding and decryption. Finally, the transmitted voice signal is regained.

\section{Theoretical Analysis of Single Relay}

The input binary data is obtained from a segment of recorded voice signal. Binary data stream are fed into a Convolutional encoder. After Convolutional encoding with redundant binary bit addition, the length of the channel encoded binary data is double. The channel encoded binary data are digitally modulated and FFT conversion. Then, the data are rearranged using STBC $[10,11]$ For the first antenna, $X_{k}^{T_{x} 1}=\left[X_{0},\left(-X_{1}\right)^{*}, X_{2},\left(-X_{3}\right)^{*} \ldots \ldots \ldots\left(-X_{K-1}\right)^{*}\right]$ and the second antenna,

$X_{k}^{T x_{2}}=\left[X_{1},\left(X_{0}\right)^{*}, X_{3},\left(X_{2}\right)^{*} \ldots \ldots \ldots X_{K-1}\right]$. Then the data sent into serial to parallel converter $(\mathrm{S} / \mathrm{P})$ with 1024 symbols $(\mathrm{K}=1024)$ is processed in each OFDM block to transform into time domain signal using inverse fast fourier transform (IFFT). The signal is passed through a discrete-time baseband channel with response $h(m)$ and additive white Gaussian noise (AWGN) $w(m)$ is added. In amplify-and-forward (AF) relay scheme, the relay receives a signal from the source; the received signal is amplified and forwarded to the destination in spite of the source-relay link quality. In Phase I, the source-destination and the source-relay received signals are transmitted.

In Phase II, the relay employs a linear precoder $\boldsymbol{F}$ on the received signal vector $r_{s, r}(m)$. The $M_{r} \times M_{r}$ precoding matrix $\mathrm{F}$ is given by [12-14]

$$
F=\sqrt{\frac{1}{\operatorname{tr}\left(H_{s, r}^{H} H_{s, r}\left(\sigma_{r}^{2} I_{M_{r}}+\frac{P_{s}}{M_{s}} H_{s, r}^{H} H_{s, r}\right) H_{r, d} H_{r, d}^{H}\right)}} H_{s, r}^{H} H_{r, d}^{H}
$$

Where, $H_{s, r}$ and $H_{r, d}$ are source to relay and relay to destination channel matrices; $\sigma_{r}^{2}$, $M_{r}$ and $M_{s}$ are noise variance at relay, no of receiving antenna at relay and no of transmitting antenna at sender respectively. Therefore, the signal transmitted by the relay is given by

$$
x_{r}(m)=F \cdot r_{s, r}(m)
$$

By combining the signals received at the destination in both phases, we obtain that $Y(k)$ is the received signal and $H$ is the channel matrix and $N$ is the AWGN for transmitted signal $X_{l}^{T_{x}}$. Under such consideration, received signal $Y(k)$ can be represented as 
$Y(k)=H X_{l}^{T x}+N$

\subsection{ZF Signal Detection}

ZF detectors reverse the channel matrix. The Zero Forcing technique nullifies the interference by the following weight matrix $[15,16]$

$W_{\text {ZF }}=\left(H^{H} H\right)^{-1} H^{H}$

Where $(.)^{H_{l}}$ denotes the Hermitian transpose operation. In other words, it inverts the effect of channel as

$x_{Z F}=W_{Z F} Y(k)$

\subsection{MMSE Signal Detection}

The MMSE detector employs a linear filter that can take into account the noise. The MMSE filter is found by minimizing the mean-square error (MSE) as [15, 16]

$W_{\text {MMSE }}=\left(H^{H} H+\sigma_{N_{k}}^{2} I\right)^{-1} H^{H}$

With a high SNIR, the MMSE detection becomes the ZF detection. Using the MMSE weight in equation (3), we obtain the following relationship

$x_{\text {MMSE }}=W_{\text {MMSE }} Y(k)$

\subsection{ZF-SIC Detection}

$H$ undergoes $Q R$ factorization as $[15,16]$

$$
H=Q R=Q\left\{\begin{array}{ll}
r_{1,1} & r_{1,2}
\end{array}\right]
$$

Where, $Q$ and $R$ are the unitary and upper triangular matrix respectively. On multiplying received signal $Y(k)$ of equation (3) by $Q^{H}$, we can write its product as

$$
\begin{aligned}
& x=Q^{H} Y(k) \\
& =R X_{l}{ }_{l}^{T x}+Q^{H} N
\end{aligned}
$$

Whereas, $Q^{H} N$ is a zero-mean complex Gaussian random vector. Since $Q^{H} N$ and $N$ have the same statistical properties, $Q^{H} N$ can be used to denote $N$. We get equation (9) as

$$
\left[\begin{array}{l}
x_{1} \\
x_{2}
\end{array}\right]=\left[\begin{array}{ll}
r_{1,1} & r_{1,2} \\
0 & r_{2,2}
\end{array}\right]\left[\begin{array}{l}
x_{l_{1}} \\
x_{l_{2}}
\end{array}\right]+\left[\begin{array}{l}
N_{1} \\
N_{2}
\end{array}\right]
$$


The detected desired signal $\hat{X}_{l_{1}}$ from the transmitting antennas can written on neglecting noise term from equation (10) as

$$
\begin{aligned}
& \hat{X}_{l_{1}}=\frac{\left\{x_{1}-r_{1,2}\left(x_{2} / r_{2,2}\right)\right\}}{r_{1,1}} \text { and } \\
& \hat{X}_{l_{2}}=\frac{x_{2}}{r_{2,2}}
\end{aligned}
$$

\subsection{MMSE-SIC Detection}

The received signal, channel matrix and noise are extended as $[15,16]$

$$
\begin{aligned}
& H_{e x}=\left[H^{T} \sqrt{\frac{N_{0}}{E_{s}}} I\right]^{T}, Y_{e x}(k)=\left[\begin{array}{ll}
Y(k)^{T} & 0^{T}
\end{array}\right] \text { and } \\
& N_{e x}=\left\lceil N^{T}-\sqrt{\frac{N_{0}}{E_{s}}} X_{l}^{T}\right]^{T}
\end{aligned}
$$

Where, $\frac{N_{0}}{E_{s}}=\frac{1}{S N R}$. On QR factorization of extended channel matrix $H_{e x}$, we get

$$
H_{e x}=Q_{e x} \cdot R_{e x}
$$

Where, $Q_{e x}$ and $R_{e x}$ represent a unitary matrix and an upper triangular matrix respectively. We assume that $Y(k), H, N, Q$ and $R$ are replaced by $Y_{e x}(k), H_{e x}, N_{e x}, Q_{e x}$ and $R_{e x}$ respectively and correspondingly the resulting system takes the following form

$$
\begin{aligned}
& x_{e x}=Q_{e x}^{H} \cdot Y_{e x}(k) \\
& =R_{e x} \cdot X_{l}^{T x}+Q_{e x}^{H} \cdot N_{e x}
\end{aligned}
$$

Neglecting $Q_{e x}^{H} N_{e x}$ term, the detected desired signal $\hat{X}_{l_{1}}$ from the transmitting antennas can be written from equation (14) as

$$
\begin{aligned}
& \hat{X}_{l_{1}}=\frac{\left\{x_{e x 1}-r_{e x 1,2}\left(x_{e x 2} / r_{e x 2,2}\right)\right\}}{r_{e x 1,1}} \text { and } \\
& \hat{X}_{l_{2}}=\frac{x_{e x 2}}{r_{e x 2,2}}
\end{aligned}
$$

\section{Results and Discussion}

This section presents and discusses on the results obtained by the computer simulation program written in MATLAB. Computer simulations have been performed to estimate the BER on voice signal transmission. The channel coding, FFT/IFFT size and CP length have been set to be 1/2 -rated Convolutional Encoder, 1024 and 103 symbols respectively.

The BER performance of STBC Encoded cooperative MIMO OFDM system for direct transmission (i.e., no relay) and single relay with direct transmission with different signal 
detection schemes ( ZF, ZF-SIC, MMSE and MMSE-SIC) are illustrated graphically in Figure 2 through Figure 5. It is noticeable that in all cases, the simulated system shows a quite satisfactory performance with single relay and direct transmission in QAM digital modulation and worst performance in QPSK. On critical observation, it is seen that the system performance in MMSE signal detection is comparatively better in comparison with ZF.

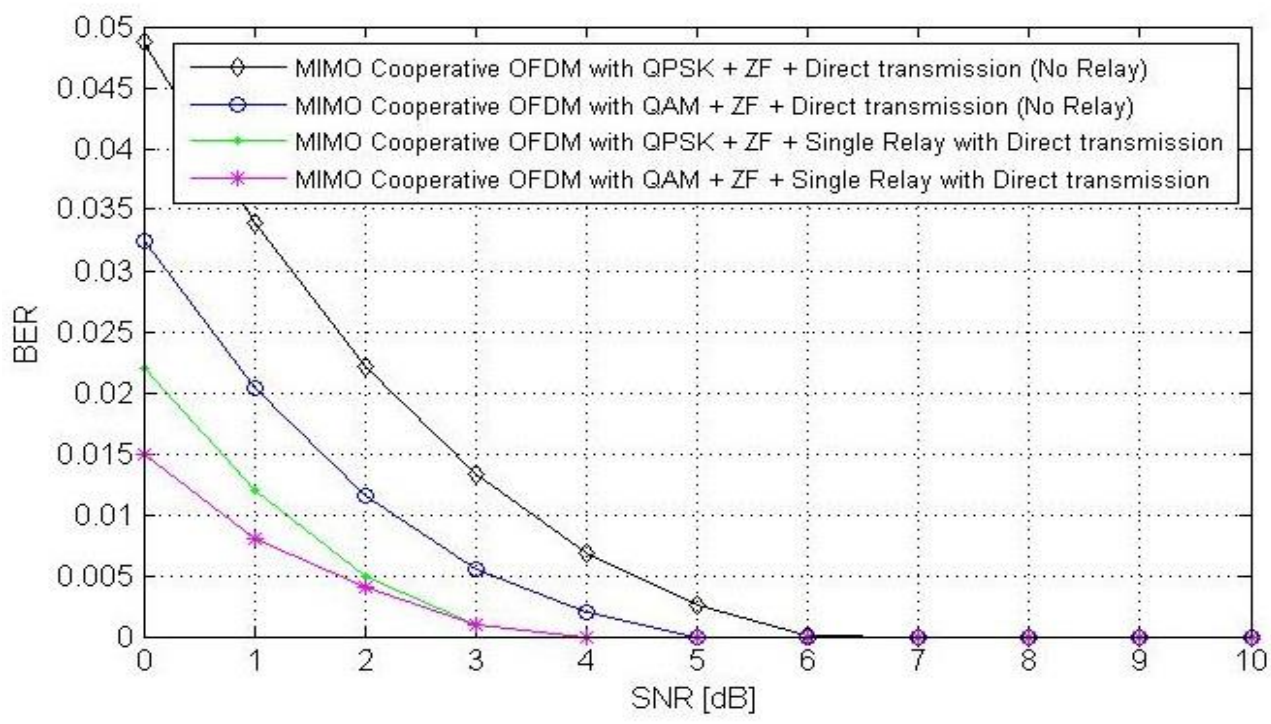

Figure 2. BER Performance of STBC Encoded Cooperative MIMO OFDM Wireless Communication System with Adaptation of ZF Signal Detection, QAM and QPSK Digital Modulation Schemes under Scenario of Direct Transmission and Single Relaying

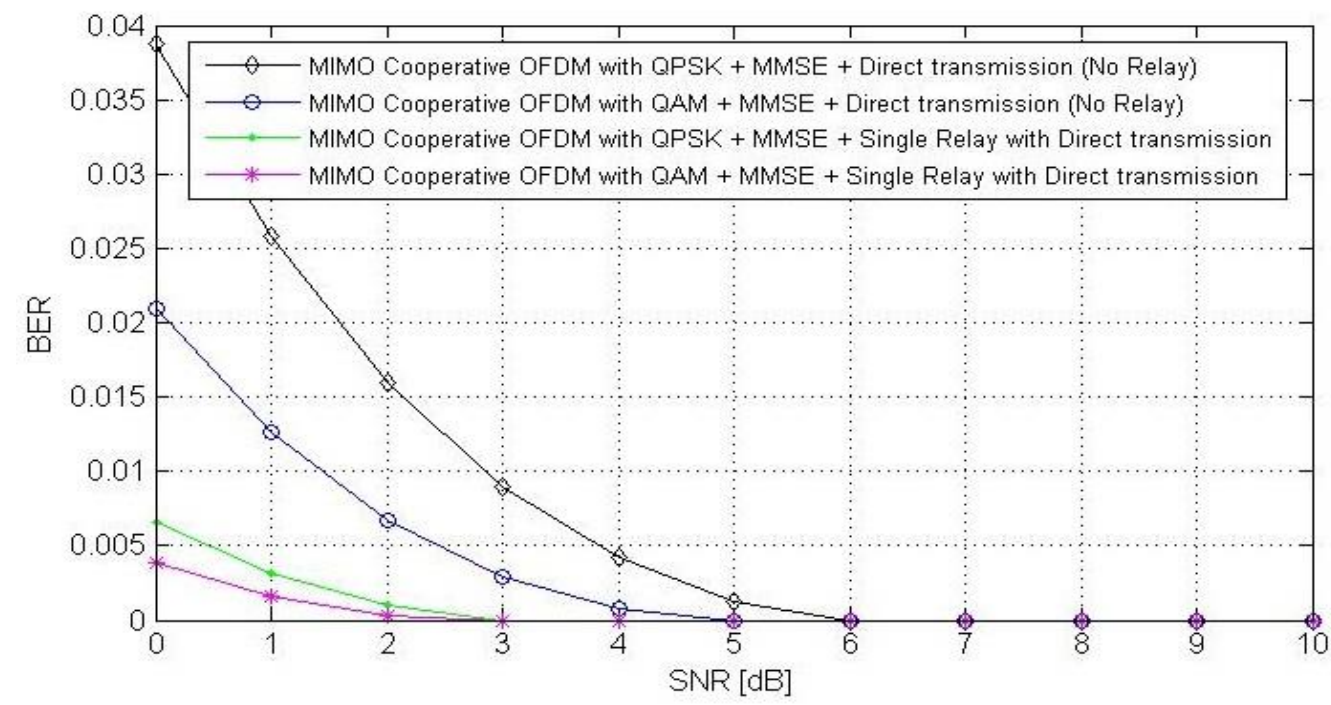

Figure 3. BER Performance of STBC Encoded Cooperative MIMO OFDM Wireless Communication System with Adaptation of MMSE Signal Detection, QAM and QPSK Digital Modulation Schemes under Scenario of Direct Transmission and Single Relaying 


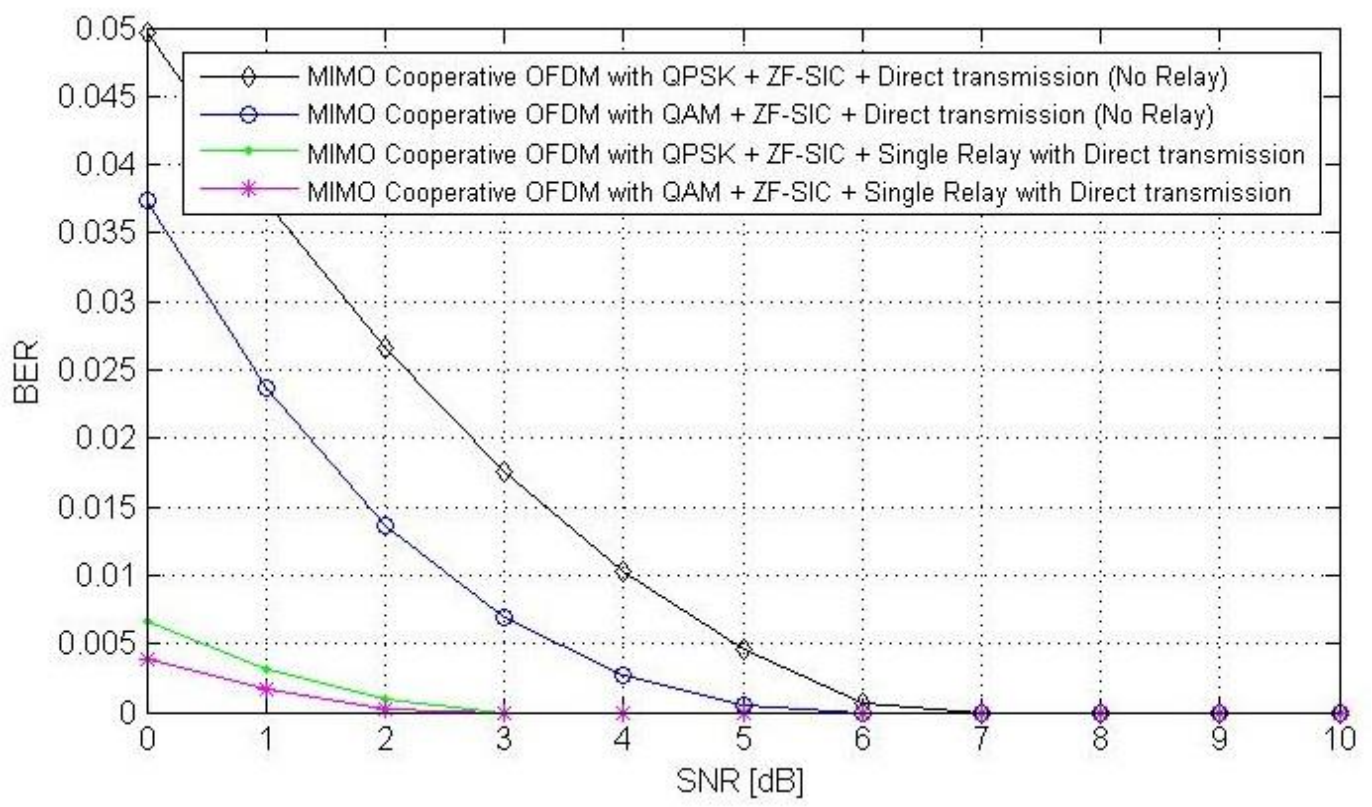

Figure 4. BER Performance of STBC Encoded Cooperative MIMO OFDM Wireless Communication System with Adaptation of ZF-SIC Signal Detection, QAM and QPSK Digital Modulation Schemes under Scenario of Direct Transmission and Single Relaying

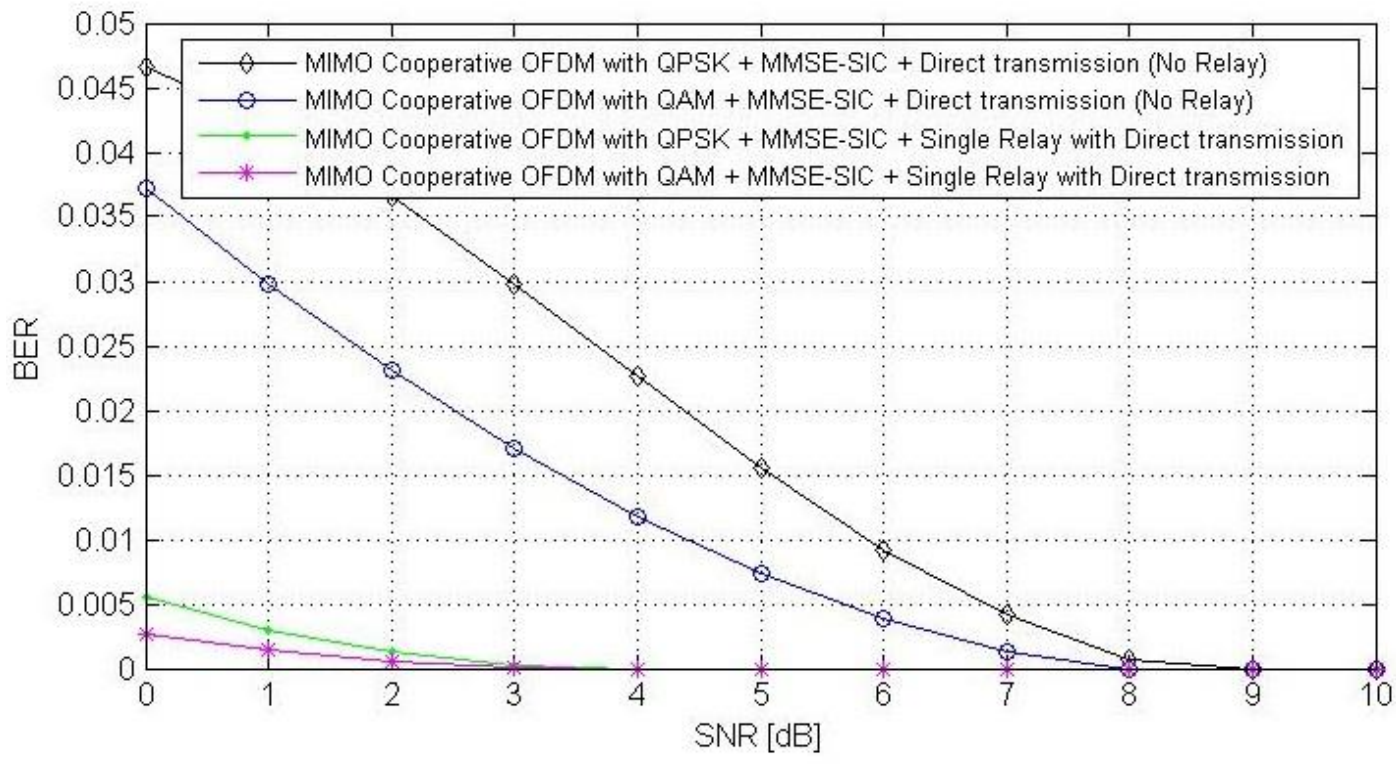

Figure 5. BER Performance of STBC Encoded Cooperative MIMO OFDM Wireless Communication System with Adaptation of MMSE-SIC Signal Detection, QAM and QPSK Digital Modulation Schemes under Scenario of Direct Transmission and Single Relaying 
In Figure 6 and 7, it is observable that retrieval of transmitted voice signal is quite satisfactory. In case of ZF-SIC and MMSE-SIC with STBC scheme implementation, the BER values are in the vicinity of acceptable level. The estimated BERs at a typically assumed SNR value of $5 \mathrm{~dB}$ for different channel equalization schemes are presented in Table 1:

Table 1. Comparison Number of Error and BER among the Four Schemes

\begin{tabular}{|l|c|c|}
\hline \multicolumn{1}{|c|}{ Scheme } & Number of Error & BER \\
\hline ZF-SIC with MIMO & 8 & $3.6169 \times 10^{-5}$ \\
\hline MMSE-SIC with MIMO & 4 & $1.8084 \times 10^{-5}$ \\
\hline ZF-SIC with STBC & 1 & $4.5211 \times 10^{-5}$ \\
\hline MMSE-SIC with STBC & 0 & 0 \\
\hline
\end{tabular}
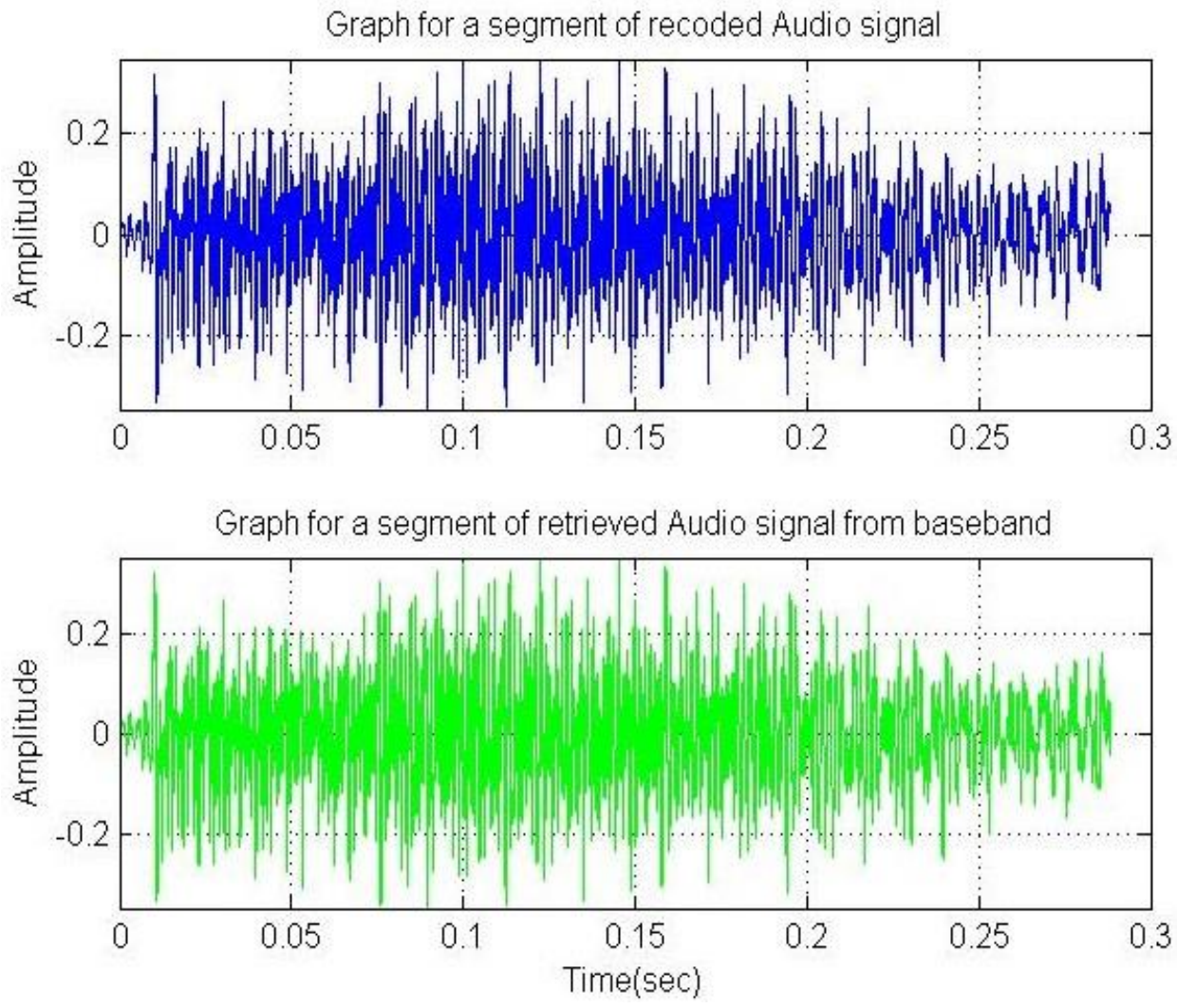

Figure 6. Graphical Illustrations Showing the Original Transmitted Voice Frequency Signal and Retrieved Signals at 5 Db With ZF-SIC and STBC Schemes 

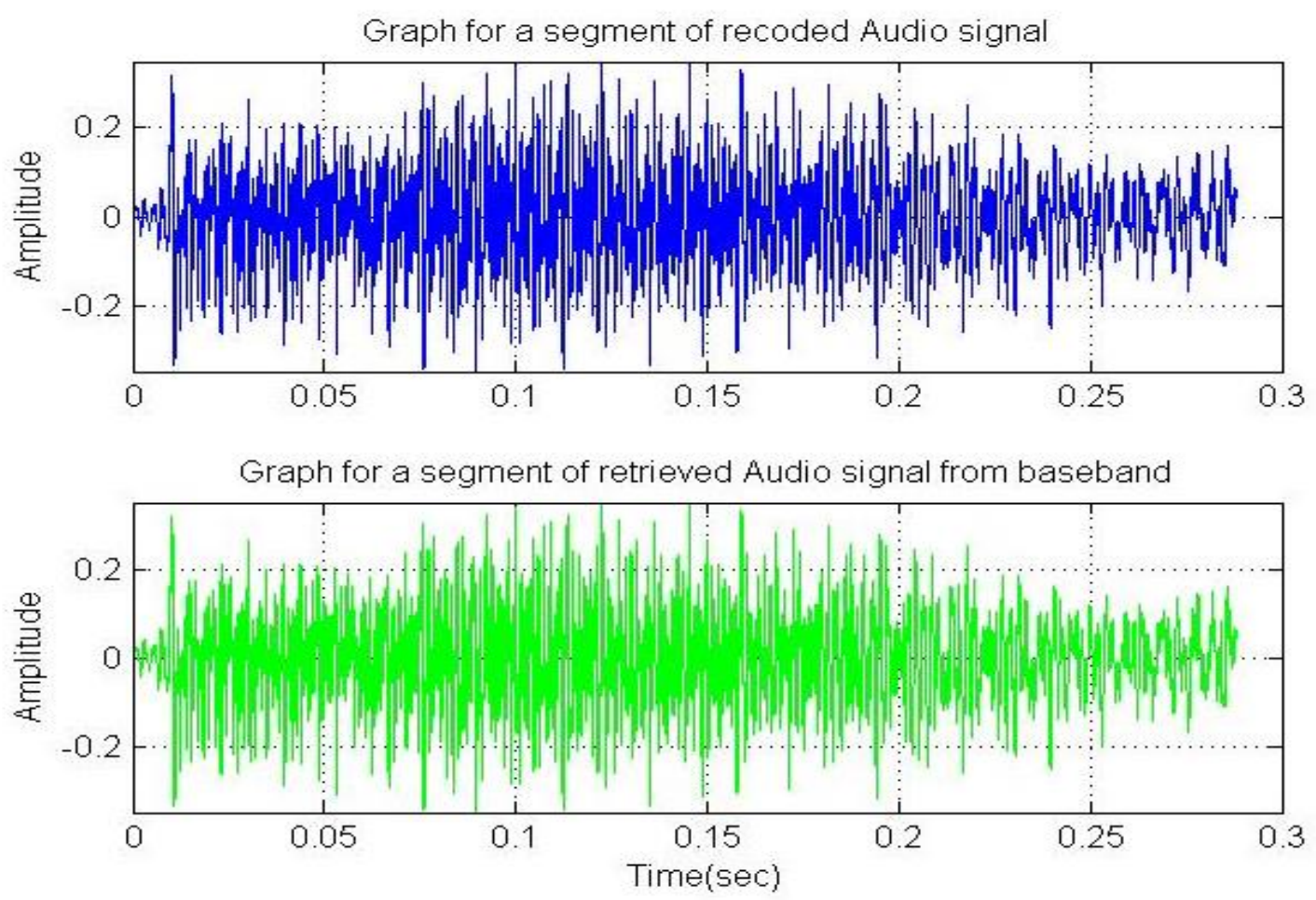

Figure 7. Graphical Illustrations Showing the Original Transmitted Voice Frequency Signal and Retrieved Signals at $5 \mathrm{~dB}$ with MMSE-SIC and STBC Schemes

\section{Conclusion}

In this paper, the scenario of enabling high quality voice transmission over STBC based MIMO Cooperative OFDM wireless communication system has been presented. In order to provide enhanced and satisfactory system performance for voice frequency signal transmission over Cooperative communication, the MMSE-SIC channel equalization scheme can be preferably implemented among the four signal detection/equalization techniques.

\section{Reference}

[1] M. Uysal, "Cooperative Communications for Improved Wireless Network Transmission: Framework for Virtual Antenna Array Applications", Information Science Reference, USA, (2009).

[2] P. Liu, Z. Tao, Z. Lin, E. Erkip and S. Panwar, "Cooperative wireless communications: A cross-layer approach", IEEE Wireless Communications, vol. 13, (2006), pp. 84-92.

[3] E. V. D. Meulen, "Three-terminal communication channels", Advances in Applied Probability, vol. 3, (1971), pp. 120-154.

[4] T. M. Cover and A. A. El Gamal, "Capacity theorems for the relay channel”, IEEE Trans. Inf. Theory, vol. 25, (1979), pp. 572-584.

[5] M. Janani, A. Hedayat, T. E. Hunter and A. Nosratinia, "Coded cooperation in wireless communications: space-time transmission and iterative decoding”, IEEE Transactions on Signal Processing, vol. 52, pp. 362371, (2004).

[6] J. N. Laneman, D. N. C. Tse and G. W. Wornell, "Cooperative Diversity in Wireless Networks: Efficient Protocols and Outage Behavior”, IEEE Trans. Inf. Theory, vol. 50, no. 12, (2004), pp. 3062-3080.

[7] D. S. Michalopoulos, H. A. Suraweera, G. K. Karagiannidis and R. Schober, "Amplify-and-Forward Relay Selection with Outdated Channel Estimates", IEEE transactions on communications, vol. 60, no. 5, (2012). 
[8] D. Haque, R. Yasmin, S. E. Ullah and M. A. F. M. R. Hasan, "Performance of a Concatenated Interleaved Forward Error Correction Scheme Based Orthogonal Frequency Division Multiplexing System”, Asian Journal of Information Technology, (2008), pp. 277-280.

[9] M. G. Rashed, M. H. Kabir, M. S. Reza, M. M Islam, R. A. Shams, S. Masum and S. E. Ullah, "Transmission of Voice Signal: BER Performance Analysis of Different FEC Schemes Based OFDM System over Various Channels", International Journal of Advanced Science and Technology, vol. 34, (2011).

[10] S. M. Alamouti, "A simple transmit diversity technique for wireless communications", IEEE Journal on Selected Areas in Communication, vol. 16, (1998), pp. 1451-1458.

[11] V. Tarokh, H. Jafarkhani and A. R. Calderbank, "Space-Time Block Coding for Wireless Communications: Performance Results, IEEE Journal on Selected Areas in Communications, vol. 17, no. 3, (1999), pp. 451460.

[12] P. U. Sripathi and J. S. Lehnert, "A throughput scaling law for a class of wireless relay networks", Proc. 38th Annual Asilomar Conference on Signals, Systems and Computers, vol. 2, (2004), pp. 1333-1337.

[13] X. Tang and Y. Hua, "Optimal waveform design for MIMO relaying", Proceedings of IEEE 6th Workshop on Signal Processing Advances in Wireless Communications, (2005), pp. 289-293.

[14] Y.-W. Peter Hong, Wan-Jen Huang and C.-C. Jay Kuo, "Cooperative Communications and Networking, Technologies and System Design", Springer publishing Company, New York, USA, (2010).

[15] Y. S. Cho, J. Kim, W. Y. Yang and C. G. Kang, "MIMO-OFDM Wireless Communications with Matlab", John Wiley and Sons (Asia) Pte Ltd, Singapore, (2010).

[16] L. Bai and J. Choi, "Low Complexity MIMO Detection, Springer Science and Business Media", LLC, New York, USA, (2012). 